\title{
sciendo
}

\section{THE INFLUENCE OF SELECTED FEED ADDITIVES ON MINERAL UTILISATION AND BONE CHARACTERISTICS IN LAYING HENS*}

\author{
Sylwester Świątkiewicz ${ }^{1 \star}$, Anna Arczewska-Włosek ${ }^{1}$, Witold Szczurek ${ }^{1}$, Jolanta Calik², \\ Józefa Krawczyk ${ }^{2}$, Damian Józefiak ${ }^{3}$ \\ ${ }^{1}$ Department of Nutrition Physiology, National Research Institute of Animal Production, \\ 32-083 Balice n. Kraków, Poland \\ ${ }^{2}$ Department of Poultry Breeding, National Research Institute of Animal Production, \\ 32-083 Balice n. Kraków, Poland \\ ${ }^{3}$ Department of Animal Nutrition and Feed Management, Poznań University of Life Sciences, \\ Wołyńska 33, 60-637 Poznań, Poland \\ •Corresponding author: s.swiatkiewicz@izoo.krakow.pl
}

\begin{abstract}
The trial with 240 caged ISA Brown laying hens was performed to evaluate the effect of selected feed additives on mineral utilisation as well as biomechanical (breaking strength, yielding load, stiffness) and geometrical (cortex thickness, cross-section area, weight, length) indices of tibia and femur bones. At 26 wks of age the layers were randomly assigned to 10 treatments with 12 replicates (cages) of two birds. In the study a $2 \times 5$ experimental scheme was used i.e. to 70 wks of age, the layers were fed isocaloric and isonitrogenous experimental diets containing reduced $(3.20 \%)$ or standard $(3.70 \%)$ Ca level. The diets with both Ca levels were either not supplemented, or supplemented with the studied feed additives i.e. sodium butyrate, probiotic bacteria, herbal extract blend and chitosan. There were no statistically significant effects of the experimental factors on the indices of the tibia bones. However, the diet with reduced $\mathrm{Ca}$ level decreased bone breaking strength, yielding load, stiffness, and mineralisation of the femur bones $(\mathrm{P}<0.05)$. The majority of used feed supplements, i.e. probiotic, herb extracts, and chitosan, increased biomechanical indices (breaking strength and yielding load) and mineralisation of the femur bones $(\mathrm{P}<0.05)$. Neither dietary Ca level nor feed additives affected dry matter, organic matter, ether extract, N-free extracts, crude fibre and ash digestibility, and $P$ retention and excretion; however, Ca excretion and retention was lower in the hens fed the diets with reduced $\mathrm{Ca}$ level $(\mathrm{P}<0.05)$. Relative Ca retention (Ca retained as \% of $\mathrm{Ca}$ intake) was improved by diet supplementation with probiotic, herb extracts and chitosan $(\mathbf{P}<\mathbf{0 . 0 5})$. In conclusion, this study has shown that decreased Ca dietary level $\mathbf{( 3 . 2 0 \% )}$ can negatively affect bone quality in layers, while probiotic, herb extracts and chitosan addition may improve the selected biomechanical indices of the femurs, irrespective of Ca dietary concentration.
\end{abstract}

Key words: laying hens, feed additives, calcium retention, bones quality

*This work was supported from the National Research Institute of Animal Production statutory activity (Research Project No. 05-019.1; Balice, Poland). 
Skeletal health is one of the most important issues in the modern egg production industry. Osteoporosis, which is the main concern relating to bones disorders in highperforming laying hens, particularly towards the end of lay, is defined as a severe decrease in mineralised structural bone in which $\mathrm{Ca}$ is mobilised from the bone in order to contribute to eggshell formation (Whitehead and Fleming, 2000; Whitehead, 2004). The repercussions of osteoporosis and skeletal weakening lead not only to performance and economic losses in egg production, but mainly are detrimental to the welfare of birds, causing acute and chronic pain and distress to the animals (Webster, 2004; Lay et al., 2011). Results of the study of Wilkins et al. (2011) showed a very high frequency of bones breakage in end-of-lay laying hens housed in a variety of system designs. Jendral et al. (2008) found that hens kept in conventional cages are particularly vulnerable to osteoporosis, exhibiting reduced bones mineral density, mass, cortical bone, area and breaking strength in comparison to birds kept in furnished cages.

Optimal mineral nutrition is one of the main factors affecting bone quality of high-performing laying hens. Intensive egg formation uses extremely high amounts of $\mathrm{Ca}$, which can increase $\mathrm{Ca}$ mobilisation from bones and negatively affect bone quality; thus a negative correlation between bone and eggshell quality in high-performing layers can be observed (Kim et al., 2012). For this reason, the supply of an adequate amount and form of dietary $\mathrm{Ca}$ is the most important nutritional factor for maintaining bone quality in hens (Olgun and Aygun, 2016). The results of some studies indicated that some feed additives, among others pre- and probiotics, organic acids and herb, extracts can, by their positive effect on intestinal health and physiology, improve mineral utilisation in poultry, which may in turn beneficially affect the mineralisation process in the organism, as well eggshell and bone quality (Świątkiewicz and Arczewska-Włosek, 2012; Abdelqader et al., 2013; Sobczak and Kozłowski, 2015; Świątkiewicz et al., 2015; Olgun, 2016; Li et al., 2017).

Therefore, the objective of this experiment was to investigate the influence of selected feed additives i.e. sodium butyrate, probiotic bacteria, herbal extract blend and chitosan in laying hens fed with standard or decreased dietary $\mathrm{Ca}$, on mineral utilisation, as well as biomechanical and geometrical indices of the tibia and femur bones in laying hens.

\section{Material and methods}

\section{Birds and experimental diets}

All experimental procedures were performed in accordance with the guidelines of the Local Krakow Ethics Committee for Experiments with Animals. A total of 240 18-wk-old ISA Brown hens, obtained from a commercial source, were placed in a poultry house, in cage on a wire-mesh floor, under controlled climate conditions. The cage dimensions were $30 \mathrm{~cm} \times 120 \mathrm{~cm} \times 50 \mathrm{~cm}\left(3600 \mathrm{~cm}^{2}\right.$ of total floor space). During the pre-experimental period, up to the hens' $26 \mathrm{wks}$ of age, the birds were fed a standard laying-hen diet ad libitum, containing $170 \mathrm{~g} / \mathrm{kg}$ crude protein, $11.6 \mathrm{MJ} / \mathrm{kg}$ $\mathrm{AME}_{\mathrm{N}}, 37.0 \mathrm{~g} / \mathrm{kg}$ calcium and $3.8 \mathrm{~g} / \mathrm{kg}$ available phosphorus. 
At wk 26, the hens were randomly allocated to one of 10 treatments and fed experimental diets until wk 70. Each treatment comprised 12 replicates of 2 hens (in one cage). During the experiment the hens were provided feed and water ad libitum, and were exposed to a $14 \mathrm{~L}: 10 \mathrm{D}$ lighting schedule, with a light intensity of 10 lux.

Table 1. Composition and nutrient content of experimental diets, $\mathrm{g} / \mathrm{kg}$ air dry matter

\begin{tabular}{lcc}
\hline \multicolumn{1}{c|}{ Item } & Reduced dietary level of Ca & Standard dietary level of Ca \\
\hline Ingredient $(\mathrm{g} / \mathrm{kg}):$ & & \\
corn & 417.1 & 423.1 \\
wheat & 240.0 & 210.0 \\
soybean meal & 230.0 & 236.0 \\
rapeseed oil & 13.0 & 19.0 \\
limestone & 78.0 & 90.0 \\
monocalcium phosphate & 12.5 & 12.5 \\
NaCl & 3.0 & 3.0 \\
DL-Methionine & 1.4 & 1.4 \\
vitamin-mineral premix ${ }^{1}$ & 5.0 & 5.0 \\
Nutrients composition: & & 11.60 \\
metabolizable energy $(\mathrm{MJ} / \mathrm{kg})^{2}$ & 11.60 & 170.0 \\
crude protein & 170.0 & 8.35 \\
Lys & 8.35 & 4.10 \\
Met & 4.10 & 37.0 \\
Ca & 32.0 & 6.15 \\
total P & 6.15 & 3.90 \\
available P & 3.90 & \\
\hline
\end{tabular}

${ }^{1}$ The premix provided per $1 \mathrm{~kg}$ of diet: vitamin $\mathrm{A}-10,000 \mathrm{IU}$; vitamin $\mathrm{D}_{3}-3,000 \mathrm{IU}$; vitamin $\mathrm{E}-50 \mathrm{IU}$; vi$\operatorname{tamin} \mathrm{K}_{3}-2 \mathrm{mg}$; vitamin $\mathrm{B}_{1}-1$; vitamin $\mathrm{B}_{2}-4 \mathrm{mg}$; vitamin $\mathrm{B}_{6}-1.5 \mathrm{mg}$; vitamin $\mathrm{B}_{12}-0.01 \mathrm{mg}$; Ca-pantothenate $8 \mathrm{mg}$; niacin - $25 \mathrm{mg}$; folic acid $-0.5 \mathrm{mg}$; choline chloride $-250 \mathrm{mg}$; manganese $-100 \mathrm{mg}$; zinc - $50 \mathrm{mg}$; iron - $50 \mathrm{mg}$; copper $-8 \mathrm{mg}$; iodine $-0.8 \mathrm{mg}$; selenium $-0.2 \mathrm{mg}$, cobalt $-0.2 \mathrm{mg}$.

${ }^{2}$ Calculated according to European Table (Janssen, 1989) as a sum of the ME content of components.

The composition of the experimental cereal-soybean diets is given in Table 1. In the study a $2 \times 5$ factorial arrangement was used, so the experimental diets contained two levels of $\mathrm{Ca}$ (reduced $-3.20 \%$ or standard $-3.70 \%$ ), and were supplemented with five experimental additives: none, sodium butyrate $(700 \mathrm{mg} / \mathrm{kg}$, GUSTOR XXI B 70, NOREL S.A., Spain), probiotic bacteria (150 mg/kg, PROTEXIN commercial preparation containing in $1 \mathrm{~g}$ : Lactobacillus plantarum $-1.26 \times 10^{7} \mathrm{cfu}$; Lactobacillus bulgaricus $-2.06 \times 10^{7} ;$ Lactobacillus acidophilus $-2.06 \times 10^{7}$; Lactobacillus rhamnosus $-2.06 \times 10^{7}$; Bifidobacterium bifidum $-2.00 \times 10^{7}$; Streptococcus thermophilus $-4.10 \times 10^{7}$; Enterococcus faecium $-5.90 \times 10^{7}$; Aspergillus oryzae -5.32 $\left.\times 10^{6}\right)$, herb extract blend $(2000 \mathrm{mg} / \mathrm{kg}$ feed, $1 \mathrm{~kg}$ of blend provided: dry extract from Echinacea purpurea, $4000 \mathrm{mg}$; oleoresin Salvia officinalis, $27800 \mathrm{mg}$; oleoresin Thymus vulgaris, $5000 \mathrm{mg}$; oil extract from Rosmarinus officinalis, $2500 \mathrm{mg}$; oil from Allium sativum, $1670 \mathrm{mg}$; and oil from Origanum vulgare, $1000 \mathrm{mg}$; Intermag Sp. z o.o., Poland), or chitosan (100 mg/kg used as Chimet-pasz preparation, Gumitex Poli-Farm, Poland). 
The nutrient content of the diets (Table 1) was calculated on the basis of the chemical composition of raw feedstuffs, and the ME value was calculated based on equations from European Tables (Janssen, 1989). The chemical composition of the feed materials was determined using AOAC (2000) methods for moisture (930.15), crude protein (984.13), crude fat (920.39), fibre (978.10) and ash (942.05). Amino acids were analysed in acid hydrolysates after initial peroxidation of sulphur amino acids by colour reaction with the ninhydrin reagent (Beckman-System Gold 126 AA Automatic Analyzer; Beckman Coulter, Inc., Pasadena, CA, US; Method 982.30; AOAC 2000). The Ca content was determined by flame atomic absorption spectrophotometry (Method 968.08; AOAC, 2000) and total P content was determined by colorimetry using the molybdo-vanadate method (Method 965.17; AOAC, 2000).

\section{Measurements}

At 40 wks of age, the digestibility coefficients of nutrients were evaluated by the total collection method. The total collection of excreta was conducted over $5 \mathrm{~d}$ and the feed consumption for each cage was recorded. Excreta was stored in plastic bags at $-20^{\circ} \mathrm{C}$ for $5 \mathrm{wks}$ and, after thawing, was dried in an oven at $50^{\circ} \mathrm{C}$ to a constant weight, weighed, and finely ground. The contents of the nutrients in the diets and excreta were estimated using the same methods as was given for the feed materials. Apparent total tract digestibility coefficient of dry matter was calculated as dry matter intake - dry matter excretion/dry matter intake. In the same way, the digestibility of organic matter, crude fat, $\mathrm{N}$-free extracts, crude fibre and ash was calculated. Calcium or $\mathrm{P}$ retention (mg) was calculated as: $\mathrm{Ca}$ or $\mathrm{P}$ intake $-\mathrm{Ca}$ or $\mathrm{P}$ excretion. Calcium or $\mathrm{P}$ relative retention (as a $\%$ of $\mathrm{Ca}$ or $\mathrm{P}$ intake) was calculated as: $\mathrm{Ca}$ or $\mathrm{P}$ intake $-(\mathrm{Ca}$ or $\mathrm{P}$ intake $-\mathrm{Ca}$ or $\mathrm{P}$ excretion $) / \mathrm{Ca}$ or $\mathrm{P}$ intake $\times 100$.

At the end of the experiment, i.e. at $70 \mathrm{wks}$ of age, all of the hens were sacrificed through cervical dislocation. The tibia and femur from both legs were collected, cleaned of soft tissues, weighed and frozen $\left(-20^{\circ} \mathrm{C}\right)$ until analysis. For determination of ash, the left tibias and toes were dried for $24 \mathrm{~h}$ at $105^{\circ} \mathrm{C}$, weighed, and dry-ashed in a muffle furnace at $600^{\circ} \mathrm{C}$. A mass of $0.2 \mathrm{~g}$ of bone ash was dissolved in $10 \mathrm{~mL}$ of $6 \mathrm{M}$ hydrochloric acid.

For measurements of the biomechanical and geometrical properties of the bones, the right tibias were used. Biomechanical properties were determined by means of the 3-point bending test (Instron 5542; Instron, Norwood, MA, US). Bone breaking strength and yielding load were measured as a graphical record from post-deformation curves. Stiffness in elastic conditions was calculated as a yielding load/elastic deformation ratio. Tibia length, cortex thickness and external and internal diameters (for cross-section area calculations) were measured at the breaking location, using an electronic slide caliper. The cross-section area was calculated from the equation: $3.14(\mathrm{HB}-\mathrm{hb}) / 4$, where $\mathrm{H}=$ external vertical diameter; $\mathrm{B}=$ external horizontal diameter; $\mathrm{h}=$ internal vertical diameter; and $\mathrm{b}=$ internal horizontal diameter.

\section{Statistical analysis}

The data were subjected to statistical analysis using a completely randomised design in accordance with the GLM procedure of Statistica 5.0 (StatSoft, Inc., Tulsa, 
OK, USA). All data were analysed using two-way ANOVA. When significant differences in treatment means were detected (ANOVA), Duncan's multiple range test was applied to separate means. Statistical significance was considered to be $\mathrm{P} \leq 0.05$.

\section{Results}

In this study, the reduced dietary level of Ca did not decrease laying performance (egg production, feed conversion ratio), but, as it was presented in our previous paper (Świątkiewicz et al., 2018), negatively affected eggshell quality in older layers. Moreover, chitosan and herb extracts had beneficial effect on laying rate and chosen indices of eggshell quality (Świątkiewicz et al., 2018).

Table 2. Effects of dietary treatments on biomechanical parameters of tibia bones

\begin{tabular}{|c|c|c|c|c|c|c|c|c|}
\hline \multirow{2}{*}{ Item } & \multirow{2}{*}{ Feed additives } & \multicolumn{3}{|c|}{ Dietary $\mathrm{Ca}$ level } & \multirow{2}{*}{ SEM } & \multicolumn{3}{|c|}{ Effect of: } \\
\hline & & Reduced & Standard & Mean & & Ca level & Additives & Interaction \\
\hline \multirow{6}{*}{$\begin{array}{l}\text { Bone } \\
\text { breaking } \\
\text { strength } \\
\text { (N) }\end{array}$} & None & 169 & 172 & 170 & 2.25 & 0.421 & 0.488 & 0.661 \\
\hline & Sodium butyrate & 173 & 176 & 174 & & & & \\
\hline & Probiotic & 170 & 175 & 173 & & & & \\
\hline & Herb extracts & 173 & 178 & 175 & & & & \\
\hline & Chitosan & 173 & 177 & 175 & & & & \\
\hline & Mean & 172 & 176 & & & & & \\
\hline \multirow{6}{*}{$\begin{array}{l}\text { Yielding } \\
\text { load }(\mathrm{N})\end{array}$} & None & 107 & 109 & 108 & 1.12 & 0.580 & 0.782 & 0.976 \\
\hline & Sodium butyrate & 109 & 110 & 109 & & & & \\
\hline & Probiotic & 107 & 112 & 109 & & & & \\
\hline & Herb extracts & 111 & 114 & 112 & & & & \\
\hline & Chitosan & 108 & 112 & 110 & & & & \\
\hline & Mean & 108 & 111 & & & & & \\
\hline \multirow{6}{*}{$\begin{array}{l}\text { Stiffness } \\
(\mathrm{N} / \mathrm{mm})\end{array}$} & None & 132 & 137 & 134 & 1.87 & 0.392 & 0.519 & 0.852 \\
\hline & Sodium butyrate & 132 & 139 & 136 & & & & \\
\hline & Probiotic & 133 & 136 & 135 & & & & \\
\hline & Herb extracts & 138 & 143 & 140 & & & & \\
\hline & Chitosan & 138 & 143 & 140 & & & & \\
\hline & Mean & 135 & 139 & & & & & \\
\hline
\end{tabular}

In our experiment there was no statistically significant influence of experimental factors (dietary $\mathrm{Ca}$ concentration, used feed additives) on the biomechanical and geometrical parameters, as well as mineralisation of the tibia bones (Tables 2 and 3). However, the femur bones in the laying hens fed the diet with reduced Ca level were characterised by significantly decreased breaking strength, yielding load, stiffness and mineralisation of femur bones (Tables 4 and 5). The majority of the used feed additives had a significant, positive influence on the biomechanical indices of the femur bones. Thus, the femurs of the laying hens fed the diet supplemented with probiotic bacteria, herb extracts, or chitosan had a significantly higher $(\mathrm{P}<0.05)$ breaking 
strength, yielding load and mineralisation than in the unsupplemented group (Tables 4 and 5). The other additive used, i.e. sodium butyrate, did not affect any of the analysed bone characteristics. Neither dietary Ca level nor diet feed additives had an effect on nutrients (dry matter, organic matter, ether extract, $\mathrm{N}$-free extracts, crude fibre and crude ash) digestibility, as well as P retention and excretion (Table 6), but $\mathrm{Ca}$ excretion and retention were significantly lower in the hens fed the diets with reduced $\mathrm{Ca}$ level (Table 7). The beneficial influence of probiotic, herb extracts and chitosan on femur bone quality was assisted by their positive effect on relative $\mathrm{Ca}$ retention (Ca retained as \% of $\mathrm{Ca}$ intake) (Table 7).

Table 3. Effects of dietary treatments on geometrical parameters and mineralization of tibia bones

\begin{tabular}{|c|c|c|c|c|c|c|c|c|}
\hline \multirow{2}{*}{ Item } & \multirow{2}{*}{ Feed additives } & \multicolumn{3}{|c|}{ Dietary Ca level } & \multirow{2}{*}{ SEM } & \multicolumn{3}{|c|}{ Effect of: } \\
\hline & & Reduced & Standard & Mean & & Ca level & Additives & Interaction \\
\hline \multirow{6}{*}{$\begin{array}{l}\text { Cortex } \\
\text { thickness } \\
(\mathrm{mm})\end{array}$} & None & 0.961 & 0.970 & 0.965 & 0.0181 & 0.719 & 0.617 & 0.564 \\
\hline & Sodium butyrate & 0.972 & 0.974 & 0.973 & & & & \\
\hline & Probiotic & 0.966 & 0.964 & 0.965 & & & & \\
\hline & Herb extracts & 0.965 & 0.975 & 0.970 & & & & \\
\hline & Chitosan & 0.964 & 0.972 & 0.968 & & & & \\
\hline & Mean & 0.966 & 0.971 & & & & & \\
\hline \multirow{6}{*}{$\begin{array}{l}\text { Cross- } \\
\text { section } \\
\text { area } \\
\left(\mathrm{mm}^{2}\right)\end{array}$} & None & 19.4 & 19.8 & 19.6 & 0.289 & 0.849 & 0.889 & 0.556 \\
\hline & Sodium butyrate & 19.8 & 20.0 & 19.9 & & & & \\
\hline & Probiotic & 19.5 & 19.5 & 19.5 & & & & \\
\hline & Herb extracts & 19.5 & 19.9 & 19.7 & & & & \\
\hline & Chitosan & 19.6 & 19.8 & 19.7 & & & & \\
\hline & Mean & 19.6 & 19.8 & & & & & \\
\hline \multirow{6}{*}{$\begin{array}{l}\text { Tibia } \\
\text { weight } \\
\text { (g) }\end{array}$} & None & 11.5 & 11.5 & 11.5 & 0.104 & 0.827 & 0.912 & 0.662 \\
\hline & Sodium butyrate & 11.5 & 11.9 & 11.7 & & & & \\
\hline & Probiotic & 11.6 & 11.5 & 11.6 & & & & \\
\hline & Herb extracts & 11.6 & 11.6 & 11.6 & & & & \\
\hline & Chitosan & 11.5 & 11.7 & 11.6 & & & & \\
\hline & Mean & 11.5 & 11.6 & & & & & \\
\hline \multirow{6}{*}{$\begin{array}{l}\text { Tibia } \\
\text { length } \\
(\mathrm{mm})\end{array}$} & None & 124 & 124 & 124 & 0.432 & 0.886 & 0.734 & 0.754 \\
\hline & Sodium butyrate & 123 & 125 & 124 & & & & \\
\hline & Probiotic & 122 & 122 & 122 & & & & \\
\hline & Herb extracts & 122 & 122 & 122 & & & & \\
\hline & Chitosan & 123 & 123 & 123 & & & & \\
\hline & Mean & 122 & 123 & & & & & \\
\hline \multirow{6}{*}{$\begin{array}{l}\text { Crude ash } \\
\text { content in } \\
\text { tibia bones } \\
(\mathrm{g} / \mathrm{kg})\end{array}$} & None & 309 & 308 & 309 & 3.861 & 0.556 & 0.675 & 0.778 \\
\hline & Sodium butyrate & 310 & 316 & 313 & & & & \\
\hline & Probiotic & 317 & 318 & 317 & & & & \\
\hline & Herb extracts & 313 & 318 & 315 & & & & \\
\hline & Chitosan & 315 & 319 & 317 & & & & \\
\hline & Mean & 313 & 316 & & & & & \\
\hline
\end{tabular}


Table 4. Effects of dietary treatments on biomechanical parameters of femur bones

\begin{tabular}{|c|c|c|c|c|c|c|c|c|}
\hline \multirow{2}{*}{ Item } & \multirow{2}{*}{ Feed additives } & \multicolumn{3}{|c|}{ Dietary Ca level } & \multirow{2}{*}{ SEM } & \multicolumn{3}{|c|}{ Effect of: } \\
\hline & & Reduced & Standard & Mean & & Ca level & Additives & Interaction \\
\hline \multirow{6}{*}{$\begin{array}{l}\text { Bone } \\
\text { breaking } \\
\text { strength } \\
\text { (N) }\end{array}$} & None & 154 & 170 & $162 \mathrm{a}$ & 3.77 & 0.007 & 0.040 & 0.437 \\
\hline & Sodium butyrate & 164 & 169 & $167 \mathrm{ab}$ & & & & \\
\hline & Probiotic & 166 & 185 & $176 \mathrm{~b}$ & & & & \\
\hline & Herb extracts & 166 & 184 & $175 \mathrm{~b}$ & & & & \\
\hline & Chitosan & 171 & 188 & $179 \mathrm{~b}$ & & & & \\
\hline & Mean & $164 a$ & $174 \mathrm{~b}$ & & & & & \\
\hline \multirow{6}{*}{$\begin{array}{l}\text { Yielding } \\
\text { load }(\mathrm{N})\end{array}$} & None & 102 & 112 & $107 \mathbf{a}$ & 1.48 & 0.006 & 0.031 & 0.919 \\
\hline & Sodium butyrate & 109 & 117 & $113 \mathrm{ab}$ & & & & \\
\hline & Probiotic & 110 & 122 & $116 \mathrm{~b}$ & & & & \\
\hline & Herb extracts & 112 & 117 & $115 \mathrm{~b}$ & & & & \\
\hline & Chitosan & 114 & 121 & $118 \mathrm{~b}$ & & & & \\
\hline & Mean & 109 a & $117 \mathrm{~b}$ & & & & & \\
\hline \multirow{6}{*}{$\begin{array}{l}\text { Stiffness } \\
(\mathrm{N} / \mathrm{mm})\end{array}$} & None & 130 & 148 & 139 & 2.37 & 0.006 & 0.164 & 0.765 \\
\hline & Sodium butyrate & 148 & 154 & 151 & & & & \\
\hline & Probiotic & 141 & 162 & 152 & & & & \\
\hline & Herb extracts & 145 & 158 & 151 & & & & \\
\hline & Chitosan & 144 & 159 & 152 & & & & \\
\hline & Mean & $142 \mathrm{a}$ & $156 \mathrm{~b}$ & & & & & \\
\hline
\end{tabular}

$\mathrm{a}, \mathrm{b}$ - the values in the rows with different letters differ significantly $(\mathrm{P} \leq 0.05)$.

Table 5. Effects of dietary treatments on geometrical parameters and mineralization of femur bones

\begin{tabular}{|c|c|c|c|c|c|c|c|c|}
\hline \multirow{2}{*}{ Item } & \multirow{2}{*}{ Feed additives } & \multicolumn{3}{|c|}{ Dietary Ca level } & \multirow{2}{*}{ SEM } & \multicolumn{3}{|c|}{ Effect of: } \\
\hline & & Reduced & Standard & Mean & & Ca level & Additives & Interaction \\
\hline 1 & 2 & 3 & 4 & 5 & 6 & \begin{tabular}{l|}
7 \\
\end{tabular} & 8 & 9 \\
\hline \multirow{6}{*}{$\begin{array}{l}\text { Cortex } \\
\text { thickness } \\
(\mathrm{mm})\end{array}$} & None & 1.008 & 1.005 & 1.007 & 0.0162 & 0.791 & 0.679 & 0.879 \\
\hline & Sodium butyrate & 1.012 & 1.022 & 1.017 & & & & \\
\hline & Probiotic & 1.005 & 1.008 & 1.007 & & & & \\
\hline & Herb extracts & 1.004 & 1.022 & 1.013 & & & & \\
\hline & Chitosan & 1.018 & 1.020 & 1.019 & & & & \\
\hline & Mean & 1.009 & 1.015 & & & & & \\
\hline \multirow{6}{*}{$\begin{array}{l}\text { Cross-section } \\
\text { area } \\
\left(\mathrm{mm}^{2}\right)\end{array}$} & None & 22.1 & 21.9 & 22.0 & 0.345 & 0.842 & 0.743 & 0.960 \\
\hline & Sodium butyrate & 22.4 & 22.7 & 22.6 & & & & \\
\hline & Probiotic & 21.8 & 22.1 & 22.0 & & & & \\
\hline & Herb extracts & 21.7 & 22.6 & 22.2 & & & & \\
\hline & Chitosan & 22.5 & 22.6 & 22.5 & & & & \\
\hline & Mean & 22.1 & 22.4 & & & & & \\
\hline \multirow{6}{*}{$\begin{array}{l}\text { Femur } \\
\text { weight (g) }\end{array}$} & None & 9.33 & 9.31 & 9.32 & 0.107 & 0.848 & 0.977 & 0.491 \\
\hline & Sodium butyrate & 9.34 & 9.38 & 9.36 & & & & \\
\hline & Probiotic & 9.40 & 9.51 & 9.46 & & & & \\
\hline & Herb extracts & 9.39 & 9.48 & 9.44 & & & & \\
\hline & Chitosan & 9.39 & 9.43 & 9.41 & & & & \\
\hline & Mean & 9.37 & 9.42 & & & & & \\
\hline
\end{tabular}


Table 5 - contd.

\begin{tabular}{l|l|r|r|r|c|c|c|c}
\hline \multicolumn{1}{c|}{2} & \multicolumn{1}{c|}{2} & 4 & 5 & 6 & 7 & 8 & 9 \\
\hline Femur & None & 87.0 & 86.6 & $\mathbf{8 6 . 8}$ & 0.290 & 0.419 & 0.953 & 0.656 \\
length (mm) & Sodium butyrate & 85.2 & 87.4 & $\mathbf{8 6 . 3}$ & & & & \\
& Probiotic & 85.9 & 86.7 & $\mathbf{8 6 . 3}$ & & & & \\
& Herb extracts & 85.9 & 86.2 & $\mathbf{8 6 . 1}$ & & & & \\
& Chitosan & 86.7 & 86.3 & $\mathbf{8 6 . 5}$ & & & & \\
& Mean & $\mathbf{8 6 . 2}$ & $\mathbf{8 6 . 6}$ & & & & & \\
Crude ash & None & 303.8 & 310.2 & $\mathbf{3 0 7 . 0 ~ a ~} 2.412$ & 0.037 & 0.036 & 0.669 \\
content & Sodium butyrate & 315.9 & 320.1 & $\mathbf{3 1 8 . 0} \mathbf{~ a b}$ & & & \\
in femur & Probiotic & 318.8 & 331.0 & $\mathbf{3 2 4 . 9} \mathbf{~ b}$ & & & \\
bones (g/kg) & Herb extracts & 319.2 & 331.1 & $\mathbf{3 2 5 . 1} \mathbf{~ b}$ & & & \\
& Chitosan & 320.2 & 328.1 & $\mathbf{3 2 4 . 1} \mathbf{~ b}$ & & & \\
& Mean & $\mathbf{3 1 5 . 6} \mathbf{3}$ & $\mathbf{3 2 4 . 1} \mathbf{~ b}$ & & & & & \\
\hline
\end{tabular}

$\mathrm{a}, \mathrm{b}$ - the values in the rows with different letters differ significantly $(\mathrm{P} \leq 0.05)$.

Table 6. Effects of dietary treatments on digestibility of nutrients (\%)

\begin{tabular}{|c|c|c|c|c|c|c|c|c|}
\hline \multirow{2}{*}{ Item } & \multirow{2}{*}{ Feed additives } & \multicolumn{3}{|c|}{ Dietary Ca level } & \multirow{2}{*}{ SEM } & \multicolumn{3}{|c|}{ Effect of: } \\
\hline & & Reduced & Standard & Mean & & Ca level & Additives & Interaction \\
\hline 1 & 2 & 3 & 4 & 5 & 6 & 7 & 8 & 9 \\
\hline \multirow[t]{6}{*}{ Dry matter } & None & 75.2 & 74.2 & 74.7 & 0.151 & 0.295 & 0.095 & 0.755 \\
\hline & Sodium butyrate & 76.0 & 75.5 & 75.7 & & & & \\
\hline & Probiotic & 75.6 & 75.9 & 75.8 & & & & \\
\hline & Herb extracts & 75.9 & 75.7 & 75.8 & & & & \\
\hline & Chitosan & 75.9 & 75.7 & 75.8 & & & & \\
\hline & Mean & 75.7 & 75.4 & & & & & \\
\hline \multirow{6}{*}{$\begin{array}{l}\text { Organic } \\
\text { matter }\end{array}$} & None & 76.4 & 75.4 & 75.9 & 0.159 & 0.399 & 0.093 & 0.725 \\
\hline & Sodium butyrate & 77.1 & 76.8 & 77.0 & & & & \\
\hline & Probiotic & 77.0 & 77.2 & 77.1 & & & & \\
\hline & Herb extracts & 77.0 & 77.2 & 77.1 & & & & \\
\hline & Chitosan & 77.2 & 76.9 & 77.1 & & & & \\
\hline & Mean & 76.9 & 76.7 & & & & & \\
\hline \multirow[t]{6}{*}{ Crude fat } & None & 56.6 & 56.4 & 56.6 & 0.423 & 0.692 & 0.951 & 0.963 \\
\hline & Sodium butyrate & 57.3 & 57.0 & 57.2 & & & & \\
\hline & Probiotic & 57.9 & 56.6 & 57.3 & & & & \\
\hline & Herb extracts & 57.2 & 57.7 & 57.5 & & & & \\
\hline & Chitosan & 57.0 & 56.5 & 56.8 & & & & \\
\hline & Mean & 57.2 & 56.9 & & & & & \\
\hline \multirow{6}{*}{$\begin{array}{l}\mathrm{N} \text {-free } \\
\text { extracts }\end{array}$} & None & 89.8 & 87.5 & 88.6 & 0.190 & 0.130 & 0.680 & 0.076 \\
\hline & Sodium butyrate & 89.3 & 89.3 & 89.3 & & & & \\
\hline & Probiotic & 89.2 & 89.2 & 89.2 & & & & \\
\hline & Herb extracts & 89.1 & 89.3 & 89.2 & & & & \\
\hline & Chitosan & 89.2 & 89.0 & 89.1 & & & & \\
\hline & Mean & 89.3 & 88.9 & & & & & \\
\hline
\end{tabular}


Table 6 - contd.

\begin{tabular}{cl|c|c|c|c|c|c|c}
\hline \multicolumn{1}{c|}{2} & 3 & 4 & 5 & 6 & 7 & 8 & 9 \\
\hline Crude fibre & None & 5.01 & 5.64 & $\mathbf{5 . 3 3}$ & 0.241 & 0.548 & 0.631 & 0.990 \\
& Sodium butyrate & 6.50 & 6.35 & $\mathbf{6 . 4 3}$ & & & & \\
& Probiotic & 5.97 & 6.89 & $\mathbf{6 . 4 3}$ & & & & \\
& Herb extracts & 6.57 & 6.94 & $\mathbf{6 . 7 6}$ & & & & \\
& Chitosan & 6.82 & 7.22 & $\mathbf{7 . 0 2}$ & & & & \\
& Mean & $\mathbf{6 . 1 8}$ & $\mathbf{6 . 6 1}$ & & & & & \\
& None & 68.0 & 68.7 & $\mathbf{6 8 . 4}$ & 0.200 & 0.317 & 0.381 & \\
& Sodium butyrate & 69.6 & 69.4 & $\mathbf{6 9 . 5}$ & & & & \\
& Probiotic & 68.9 & 69.9 & $\mathbf{6 9 . 5}$ & & & & \\
& Herb extracts & 69.7 & 69.1 & $\mathbf{6 9 . 4}$ & & & & \\
& Chitosan & 68.7 & 69.8 & $\mathbf{6 9 . 3}$ & & & & \\
& Mean & $\mathbf{6 9 . 0}$ & $\mathbf{6 9 . 4}$ & & & & & \\
\hline
\end{tabular}

Table 7. Effects of dietary treatments on balance of calcium and phosphorus (\%)

\begin{tabular}{|c|c|c|c|c|c|c|c|c|}
\hline \multirow{2}{*}{ Item } & \multirow{2}{*}{ Feed additives } & \multicolumn{3}{|c|}{ Dietary Ca level } & \multirow{2}{*}{ SEM } & \multicolumn{3}{|c|}{ Effect of: } \\
\hline & & Reduced & Standard & Mean & & Ca level & Additives & Interaction \\
\hline 1 & 2 & 3 & 4 & 5 & 6 & 7 & 8 & 9 \\
\hline \multirow{6}{*}{$\begin{array}{l}\text { Ca excretion } \\
\text { (mg/hen per day) }\end{array}$} & None & 1309 & 1589 & $1490 \mathrm{~b}$ & 15.1 & 0.001 & 0.046 & 0.588 \\
\hline & Sodium butyrate & 1364 & 1467 & 1416 a & & & & \\
\hline & Probiotic & 1361 & 1508 & $1435 \mathrm{ab}$ & & & & \\
\hline & Herb extracts & 1311 & 1491 & 1401 a & & & & \\
\hline & Chitosan & 1337 & 1528 & $1433 \mathrm{ab}$ & & & & \\
\hline & Mean & 1353 a & $1517 \mathrm{~b}$ & & & & & \\
\hline \multirow{6}{*}{$\begin{array}{l}\text { Ca retention } \\
\text { ( } \mathrm{mg} / \text { hen per day) }\end{array}$} & None & 2809 & 3395 & 3102 & 26.4 & 0.001 & 0.250 & 0.322 \\
\hline & Sodium butyrate & 2955 & 3278 & 3116 & & & & \\
\hline & Probiotic & 3053 & 3415 & 3233 & & & & \\
\hline & Herb extracts & 2994 & 3433 & 3213 & & & & \\
\hline & Chitosan & 2923 & 3436 & 3180 & & & & \\
\hline & Mean & 2947 a & 3391 b & & & & & \\
\hline \multirow{6}{*}{$\begin{array}{l}\text { Ca retained } \\
(\% \text { of } \mathrm{Ca} \text { intake })\end{array}$} & None & 66.9 & 68.0 & $67.5 \mathrm{a}$ & 0.265 & 0.237 & 0.038 & 0.975 \\
\hline & Sodium butyrate & 68.4 & 69.1 & $68.7 \mathrm{ab}$ & & & & \\
\hline & Probiotic & 69.1 & 69.3 & $69.2 \mathrm{~b}$ & & & & \\
\hline & Herb extracts & 69.5 & 69.7 & $69.6 \mathrm{~b}$ & & & & \\
\hline & Chitosan & 69.4 & 69.8 & $69.6 \mathrm{~b}$ & & & & \\
\hline & Mean & 68.7 & 69.2 & & & & & \\
\hline \multirow{6}{*}{$\begin{array}{l}\text { P excretion } \\
\text { ( } \mathrm{mg} / \text { hen per day) }\end{array}$} & None & 653 & 670 & 662 & 3.55 & 0.084 & 0.313 & 0.061 \\
\hline & Sodium butyrate & 662 & 619 & 640 & & & & \\
\hline & Probiotic & 674 & 638 & 656 & & & & \\
\hline & Herb extracts & 649 & 652 & 650 & & & & \\
\hline & Chitosan & 654 & 656 & 655 & & & & \\
\hline & Mean & 658 & 647 & & & & & \\
\hline
\end{tabular}


Table $7-\operatorname{cotd}$.

\begin{tabular}{|c|c|c|c|c|c|c|c|c|}
\hline 1 & 2 & 3 & 4 & 5 & 6 & 7 & 8 & 9 \\
\hline \multirow{6}{*}{$\begin{array}{l}\text { P retention } \\
\text { (mg/hen per day) }\end{array}$} & None & 199 & 204 & 201 & 3.68 & 0.976 & 0.420 & 0.931 \\
\hline & Sodium butyrate & 214 & 214 & 214 & & & & \\
\hline & Probiotic & 221 & 225 & 223 & & & & \\
\hline & Herb extracts & 224 & 212 & 218 & & & & \\
\hline & Chitosan & 210 & 215 & 212 & & & & \\
\hline & Mean & 214 & 214 & & & & & \\
\hline \multirow{6}{*}{$\begin{array}{l}\mathrm{P} \text { retained } \\
(\% \mathrm{P} \text { intake })\end{array}$} & None & 23.3 & 23.4 & 23.3 & 0.342 & 0.579 & 0.407 & 0.817 \\
\hline & Sodium butyrate & 24.4 & 25.6 & 25.0 & & & & \\
\hline & Probiotic & 24.6 & 26.0 & 25.3 & & & & \\
\hline & Herb extracts & 25.5 & 24.5 & 25.0 & & & & \\
\hline & Chitosan & 24.3 & 24.7 & 24.5 & & & & \\
\hline & Mean & 24.4 & 24.8 & & & & & \\
\hline
\end{tabular}

$\mathrm{a}, \mathrm{b}$ - the values in the rows with different letters differ significantly $(\mathrm{P} \leq 0.05)$.

\section{Discussion}

To date, the amount of published data from poultry studies on the effects of feed additives on bone quality is rather limited. Similarly to results of this experiment, Świątkiewicz et al. (2014 a) observed a positive effect of probiotic bacteria (L. salivarius) on some bone quality indices in layers fed a diet with a high level of DDGS. Abdelqader et al. (2013) found increased tibia weight, density and ash concentration in aged laying hens (64-74 wks of age) fed a diet supplemented with Bacillus subtilis probiotic. Corresponding results were reported by Mutus et al. (2006) in broilers fed diets supplemented with Bacillus probiotic bacteria. They observed the positive influence of probiotic bacteria on several indices of tibia bones i.e. the thickness of the medial and lateral walls, the tibiotarsal index, percentage of ash and P content. Angel et al. (2005) found enhanced bones mineralisation and breaking strength along with increased retention of $\mathrm{Ca}$ and $\mathrm{P}$ in chickens fed a diet supplemented with Lactobacillus probiotic.

In the experiment by Houshmand et al. (2011), the bones indices of broilers were negatively affected by a low $\mathrm{Ca}$ diet; however, the addition of probiotic bacteria had a beneficial influence on these parameters and helped to overcome the problems related to a low Ca dietary level. Such a positive effect of dietary probiotic on breaking strength and mineralisation of bones was attributed by the authors to the increased retention of $\mathrm{Ca}$ in the bones (Panda et al., 2008). As it was discussed by ScholzAhrens et al. (2007), the mechanism of the beneficial influence of probiotic bacteria in terms of bones indices could probably be linked to their positive effect on mineral utilisation, which can be attributed in turn to increased solubility of minerals due to the bacteria's increased production of short-chain fatty acids, alteration of intestinal mucosa and increase of the absorption surface through the beneficial effect of bacterial fermentation products on the proliferation of enterocytes, increased expression 
of Ca-binding proteins, release of bone modulating factors, degradation of phytates by probiotic bacteria enzymes and overall improvement of gut health.

Similarly to the beneficial effect of herb extracts on selected femur characteristics found in this study, Zhou et al. (2009) reported improved breaking strength, weight and radiographic densities of the humerus, tibia and femur in older laying hens fed a diet supplemented with traditional Chinese herbs mixture (Epimedii, Rhizoma Drynariae, Rhizoma Atractylodis and Radix Astragali). The authors speculated that the mechanism of such a positive effect of herbs dietary supplementation was probably related to their action minimising structural bone loss and stimulating bone mineral absorption in osteoporotic laying hens (Zhou et al., 2009). The positive effect of herb extracts on bone quality can also be due to their beneficial influence on Ca utilisation, as was observed in this experiment and in the study performed on breeder quails (Olgun and Yildiz, 2014). More recently, Olgun (2016) demonstrated the positive effect of herb extract blend used at a low supplementation level (25 or $50 \mathrm{mg} / \mathrm{kg}$ ) on the biomechanical indices of bones in laying hens, which was assisted with increased $\mathrm{Ca}$ content; however, the shear force and shear stress of bones was reduced for layers fed the diet with a high level $(600 \mathrm{mg} / \mathrm{kg})$ of extracts. Correspondingly, Mühlbauer et al. (2003) found in a model rats study that essential oils and monoterpenes of selected herbs (sage, rosemary and thyme) are efficient inhibitors of bone resorption. The above mentioned effects of herb extracts and essential oils can be probably related to their beneficial effects on gut health, for instance on intestinal microbiota and morphology, as it was recently reported in broilers fed the diet supplemented with herb products (Giannenas et al., 2016; Kiczorowska et al., 2016). Recently, however, Leskovec et al. (2018) did not find any positive effect of plant extracts (marigold, olive leave extracts) on mineral utilization and bone characteristics in broiler chickens.

The positive effect of chitosan on some tibia bones indices, as observed in our study, could probably be explained by improved Ca digestibility. To date, the experimental data on the effect of dietary chitosan on bone quality in laying hens are very limited. Corresponding results were found in broiler chickens by Huang et al. (2005) and Świątkiewicz et al. (2014b), who observed that diet supplementation with chitosan $(0.015 \%)$ increased the digestibility of dry matter, $\mathrm{N}, \mathrm{Ca}$, and P and, as a result of improved nutrients digestibility, growth performance indices; however, these effects were not reflected in the bones characteristics. The mechanism of the beneficial effect of chitosan addition and, in this way, on tibia characteristics as observed in our study, can probably be attributed to its positive influence on intestinal morphology (Khambualai et al., 2008; Khambualai et al., 2009). Such effect was recently confirmed in Leiothrix lutea birds, where diet supplementation with chitosan $(0.5 \%)$ increased the apparent digestibility of most nutrients, improved intestinal histology, i.e. increased the villous height of the duodenum and ileum, as well as enhanced activity of intestinal enzymes (Le et al., 2015).

\section{Conclusions}

In conclusion, the findings of this study show that reducing $\mathrm{Ca}$ dietary level below $3.70 \%$ can negatively affect bone quality in high-producing laying hens, while 
probiotic, herb extracts, or chitosan addition may improve the selected biomechanical indices of bone quality in aged, high-producing laying hens, irrespective of $\mathrm{Ca}$ dietary level.

\section{Competing interests}

The authors confirm that this work has no conflict of interest.

\section{References}

A bd e l qader A., I rshaid R., A l-F a t a ft a h A.R. (2013). Effects of dietary probiotic inclusion on performance, eggshell quality, cecal microflora composition, and tibia traits of laying hens in the late phase of production. Trop. Anim. Health Prod., 45: 1017-1024.

A nge l R., D alloul R.A., D o err J. (2005). Performance of broiler chickens fed diets supplemented with a direct-fed microbial. Poultry Sci., 84: 1222-1231.

AOAC (2000). Official Methods of Analysis, Association of Official Analytical Chemists. 17th Edition. Gaithersburg (USA).

Giannen as I., Tzora A., S arakatsianos I., K aramoutsios A., Skoufos S., P apaioannou N., A nastasiou I., Skoufos I. (2016). The effectiveness of the use of oregano and laurel essential oils in chicken feeding. Ann. Anim. Sci., 16: 779-796.

Houshmand M., Azhar K., Zulkifli I., B ejo M.H., Meimandipour A., Kamyab A. (2011). Effects of non-antibiotic feed additives on performance, tibial dyschondroplasia incidence and tibia characteristics of broilers fed low-calcium diets. J. Anim. Physiol. Anim. Nutr., 95: 351-358.

H u a ng R.L., Y in Y.L., W u G.Y., Z h ang Y.G., L i T.J., Li L.L., L i M.X., T a ng Z.R., Z hang J., W a n g B., He J.H., N i e X.Z. (2005). Effect of dietary oligochitosan supplementation on ileal digestibility of nutrients and performance in broilers. Poultry Sci., 84: 1383-1388.

J a n s s e n W.M.M.A. (1989). European Table of Energy Values for Poultry Feedstuffs. 3rd ed. Working Group No. 2 of the European Branch, World's Poultry Science Association, Beekbergen, The Netherlands.

Jendral M.J., Korver D.R., Church J.S., Feddes J.J.R. (2008). Bone mineral density and breaking strength of White Leghorns housed in conventional, modified, and commercially available colony battery cages. Poultry Sci., 87: 828-837.

Khambualai O., Yamauch i K.E., Tangtaweewipat S., Cheva-I sarakul B. (2008). Effects of dietary chitosan diets on growth performance in broiler chickens. J. Poultry Sci., 45: 206-209.

Khambualai O., Yamauchi K., Tangtaweewipat T., Cheva-Isarakul B. (2009). Growth performance and intestinal histology in broiler chickens fed with dietary chitosan. British Poultry Sci., 50: 592-597.

Ki c zorowska B., S a molińska W., A l- Yas iry A.R.M., Kowalczyk-Pecka D. (2016). Effect of Boswellia serrata dietary supplementation on growth performance, gastrointestinal microflora, and morphology of broilers. Ann. Anim. Sci., 16: 835-849.

K i m W.K., B lo o m field S.A., S u gi y a ma T., R i cke S.C. (2012). Concepts and methods to understanding the bone metabolism in laying hens. World. Poultry Sci. J., 68: 71-82.

Lay D.C., Fulton R.M., Hester P.Y., Karcher D.M., Kjaer J.B., Mench J.A., Mulens B.A., Newberry R.C., Nicol C.J., O'Sullivan N.P., Porter R.E. (2011). Hen welfare in different housing systems. Poultry Sci., 90: 278-294.

Le W., Ting-Ting L., X i-Long W., Gui-P ing Y., Shi-B in Y. (2015). Chitosan supplementation may improve the digestive physiology and health of captive Leiothrix lutea. Avian Biol. Res., 8: 221-226.

Leskovec J., Levart A., Žgur S., Jordan D., P irman T., S a lobir J., Rezar V. (2018). Effects of olive leaf and marigold extracts on the utilization of nutrients and on bone mineralization using two different oil sources in broilers. J. Poult. Sci., 55: 17-27. 
Li D.D., D ing X.M., Z ha ng K.Y., B a i S.P., Wang J.P., Z e n g Q.F., S u Z.W., K a n g L. (2017). Effects of dietary xylooligosaccharides on the performance, egg quality, nutrient digestibility and plasma parameters of laying hens. Anim. Feed Sci. Technol., 225: 20-26.

Mühlbauer R.C., L ozano A., Pala cio S., Rein 1 A., F elix R. (2003). Common herbs, essential oils, and monoterpenes potently modulate bone metabolism. Bone, 32: 372-380.

Mutus R., K o c a b a g li N., A l p M., A c a r N., Era n M., G e ze n S. (2006). The effect of dietary probiotic supplementation on tibial bone characteristics and strength in broilers. Poultry Sci., 85: $1621-1625$.

O 1 g u n O. (2016). The effect of dietary essential oil mixture supplementation on performance, egg quality and bone characteristics in laying hens. Ann. Anim. Sci., 16: 1115-1125.

Olgun O., Aygun A. (2016). Nutritional factors affecting the breaking strength in laying hens. World. Poultry Sci. J., 72: 821-832.

O 1 g u n O., Y i l d i z A.Ö. (2014). Effect of dietary supplementation of essential oils mixture on performance, eggshell quality, hatchability, and mineral excretion in quail breeders. Environm. Sci. Pollut. Res., 21: 13434-13439.

P a n d a A.K., R a m a R a o S.V., R a j u M.V.L.N., S h a r m a S.R. (2008). Effect of probiotic (Lactobacillus sporogenes) feeding on egg production and quality, yolk cholesterol and humoral immune response of White Leghorn layer breeders. J. Sci. Food Agricult., 88: 43-47.

Scholz-Ahrens K.E., Ade P., Marten B., Weber P., Timm W., Asil Y., Gluer C.C., S chrezen meir J. (2007). Prebiotics, probiotics, and synbiotics affect mineral absorption, bone mineral content, and bone structure. J. Nutr., 137: 838S-846S.

S o b c zak A., K o złow s ki K. (2015). The effect of a probiotic preparation containing Bacillus subtilis ATCC PTA-6737 on egg production and physiological parameters of laying hens. Ann. Anim. Sci., 15: 711-723.

Świ ątkiewicz S., A rczewska-Włos ek A. (2012). Prebiotic fructans and organic acids as feed additives improving mineral availability. World. Poultry Sci. J., 68: 269-279.

Św i ą tki ew icz S., A rczews ka-Włosek A., Jozefiak D. (2014 a). Bones quality indices in laying hens fed diets with a high level of DDGS and supplemented with selected feed additives. Czech J. Anim. Sci., 59: 61-68.

Św i ą tki ewicz S., A rczews ka - Włos ek A., Jozefiak D. (2014 b). Feed enzymes, probiotic, or chitosan can improve the nutritional efficacy of broiler chicken diets containing a high level of distillers dried grains with solubles (DDGS). Livest. Sci., 163: 110-119.

Św i ątki ewicz S., A r c zew ska - Włosek A., Krawczyk J., P u chała M., Józe fiak D. (2015). Dietary factors improving eggshell quality: an updated review with special emphasis on microelements and feed additives. World. Poultry Sci. J., 71: 83-94.

Świątkiewicz S., Arczewska-Włosek A., Krawczyk J., Szczurek W., Puchała M., Jó zefia k D. (2018). Effect of selected feed additives on egg performance and eggshell quality in laying hens fed a diet with standard or decreased calcium content. Ann. Anim. Sci., 18: $167-183$.

We b s te r A.B. (2004). Welfare implications of avian osteoporosis. Poultry Sci., 83: 184-192.

W h i t e h e a d C.C.(2004). Overview of bone biology in egg-laying hen. Poultry Sci., 83: 193-199.

W h i t e h e a d C.C., F 1 e m ing R.H. (2000). Osteoporosis in laying hens. Poultry Sci., 79: 1033-1041.

Wilkins L.J., McKinstry J.L., Avery N.C., Knowles T.G., Brown S.N., Tarlton J., $\mathrm{N}$ i c o 1 C.J. (2011). Influence of housing system and design on bone strength and keel bone fractures in laying hens. Vet. Rec., 169: 414-420.

Z h o u Z.L., D e n g Y.F., T a o Q.S., H u Y.F., H o u J.F. (2009). Effects of Gushukang, a Chinese herbal medicine, on bone characteristics and osteoporosis in laying hens. Poultry Sci., 88: 2342-2345.

Received: 12 III 2018

Accepted: 21 III 2018 